\title{
Design and Manufacturing of the Wendelstein 7-X Cryo-Vacuum Pump
}

\author{
G. Ehrke*, a, B. Mendelevitch ${ }^{\mathrm{b}}$, J. Boscary ${ }^{\mathrm{b}}$, C. Li ${ }^{\mathrm{b}}$, O. Sellmeier ${ }^{\mathrm{b}}$, R. Stadler ${ }^{\mathrm{b}}$ \\ P. McNeely ${ }^{\mathrm{a}}$, F. Schauer ${ }^{\mathrm{b}}$ and the W7-X Team ${ }^{\mathrm{a}, \mathrm{b}}$ \\ ${ }^{a}$ Max Planck Institute for Plasma Physics, Greifswald, Germany \\ ${ }^{b}$ Max Planck Institute for Plasma Physics, Garching, Germany \\ *Corresponding author: gunnar.ehrke@ipp.mpg.de
}

The cryo-vacuum pump (CVP) system, consisting of 10 units distributed symmetrically inside the Wendelstein 7-X plasma vessel, will be installed together with the 10 units of the actively cooled high heat flux divertor. One pump each is located below the corresponding divertor, and positioned as close as possible to the flux line strike points in order to allow efficient control of plasma density, and for screening impurities. Each CVP is divided into two parts, interconnected by a transfer line, to ensure access for divertor diagnostic integration. The CVP panels are operated with supercritical helium at 3.3-3.8 K to pump discharge gases such as $\mathrm{H}_{2}$ and $\mathrm{D}_{2}$. They are protected against thermal radiation by black-oxide finished stainless steel chevrons cooled by liquid nitrogen at $77 \mathrm{~K}$. In front of this $\mathrm{LN}_{2}$-shield is a water-baffle which protects the CVP against plasma and ECRH stray radiation. It consists of copper chevrons with zero overlap, mounted on a water-cooled steel pipe. These chevrons are coated with an $\mathrm{Al}_{2} \mathrm{O}_{3}-\mathrm{TiO}_{2}$ layer. In addition, an uncooled copper shield covers the gap between waterbaffle and $\mathrm{LN}_{2}$ chevrons in order to prevent stray radiation to take this path to the He-cooled panel. The cryogenic fluids are supplied via a dedicated port plug-in which is thermally insulated by a $\mathrm{LN}_{2}$-cooled cryo-shield and superinsulation. All 10 CVPs are already manufactured and successfully leak tested under hot and cold conditions in the workshops of IPP Garching.

This paper presents the design and the manufacturing technology of the CVPs and the adjacent periphery. Results of the quality assessment such as integral He leak testing at $160{ }^{\circ} \mathrm{C}$ and cryogenic temperatures $(77 \mathrm{~K})$ are also discussed.

Keywords: Stellarator, Cryo-vacuum Pump, Cryogenic Temperature, Liquid Nitrogen

\section{Introduction}

The stellarator Wendelstein 7-X (W7-X) has been designed to operate stationary long plasma pulses up to 30 min with $10 \mathrm{MW}$ input electron-cyclotron resonance heating $(\mathrm{ECRH})$ power. The maximum available input power with the additional systems neutral beam injection (NBI) and ion-cyclotron resonance heating (ICRH) is up to $20 \mathrm{MW}$ for $10 \mathrm{~s}$ [1]. To achieve stable operating conditions it is essential to confine the energy and particles in a steady and pure plasma, and to transport the heating power out of the machine $[1,2]$. An actively water-cooled plasma wall jacket consisting of different types of plasma facing components (PFCs) and a high heat flux (HHF) divertor remove the heat loads from the plasma and controls the plasma power and the particle exhaust [3, 4]. The pumping system is essential to achieve long pulse plasma operation. The W7-X pumping system consists of two independent systems: the turbo molecular pumps (TMPs) and cryo-vacuum pumps (CVPs). The CVP allows an efficient control of high plasma densities by removing impurities and neutrals from the confinement area, and by capturing discharge gases such as Hydrogen and Deuterium [5]. The CVP contributes to keeping the plasma free of undesirable perturbations as well as to producing a vacuum in the divertor chamber of up to $10^{-3}$ mbar Hydrogen.

This paper describes the design and the manufacturing of the $10 \mathrm{~W} 7-\mathrm{X}$ CVPs that are already manufactured and successfully tested on site at IPP Garching.

\section{Design}

The CVP is one of the HHF-divertor components and is located on the backside of the corresponding horizontal HHF-divertor. Further HHF-divertor components are: horizontal and vertical divertor modules (TM-h/v), the baffle area to guide the neutral particles to the pumping gap, and the closure panels that border the divertor chamber in the poloidal (PDA) and toroidal (TDA) directions. Together these components constitute the divertor chamber (Fig. 1).

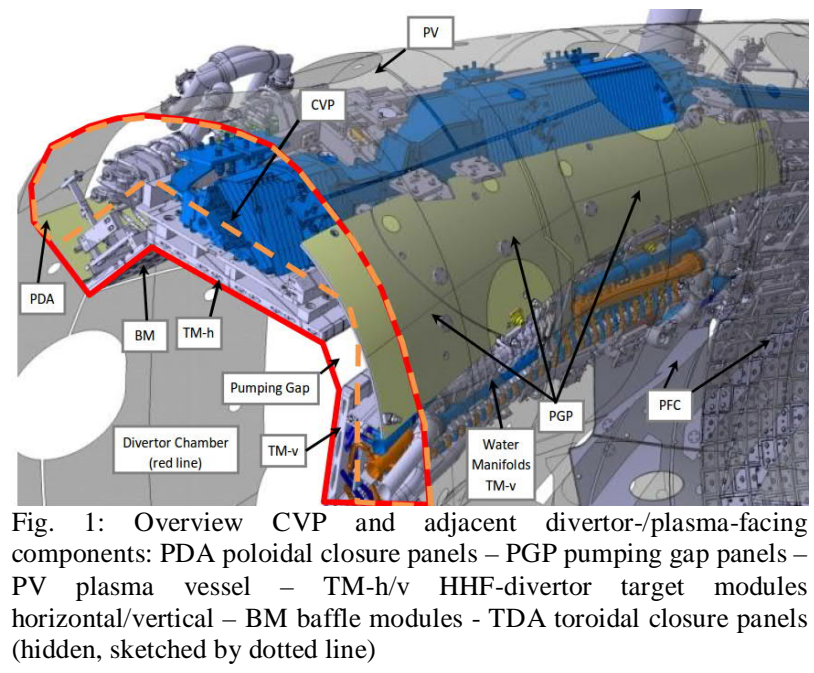

The divertor chamber is open towards the plasma mainly via the pumping gap. The PV at the opposite side of the chamber is protected by overlapping actively watercooled stainless steel pumping gap panels. 
The CVP is cooled by supercritical Helium ( $\mathrm{ScHe}$ ) such that the panel surface temperature keeps the saturation pressure equal to or below the desired vacuum pressure in the divertor chamber [6]. Fig. 3 shows the saturation curves for some $\mathrm{W} 7-\mathrm{X}$ relevant gases $\left(\mathrm{H}_{2}, \mathrm{D}_{2}\right)$. This figure also shows that He cannot be pumped by the W7-X CVP due to the operating temperatures of the ScHe-panels of 3.3 K - 3.8 K. Special scenarios with Ar-frosting need to be developed for He.

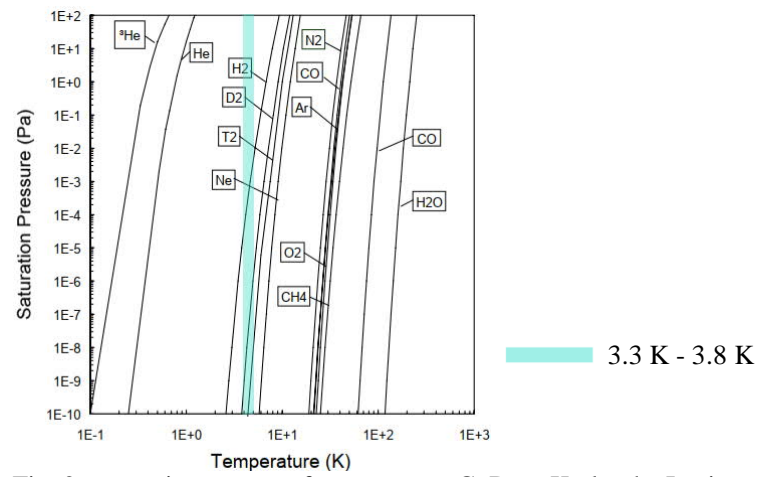

Fig. 2: saturation curves of some gases, C. Day, Karlsruhe Institute of Technology (KIT) [6]

Particles are adsorbed at the CVP panel surface that consists of porous material with high sorption capacity, and stay attached there by intermolecular forces (Van-derWaals-Forces) [6]. The layers of adsorbed gas molecules have to be pumped out by the TMPs during regeneration in order to avoid undesirable concentrations of adsorbed gas particles (allowed layer thickness on the ScHe-panel $<1 \mathrm{~mm}$ ). The regeneration also ensures a sufficient gas heat conduction between the plasma vessel and the CVP panels.

The CVP is designed to pump at least $100 \mathrm{mbar} * 1 / \mathrm{s}$ neutral gas that corresponds to the gas flow of two neutral beam injectors with $10 \mathrm{MW}$ heating power each. A safety factor of two led to a specified value of $200 \mathrm{mbar} * \mathrm{l} / \mathrm{s}$ pumped gas flow.

\section{Manufacturing process}

\subsection{ScHe-panels}

The ScHe-panel represents the functional heart of the W7-X CVP where the undesired gases are adsorbed and pumped. The adjacent peripheral sub-assemblies of the CVP have only secondary tasks: they have to transport the cryogenic media, and to protect the ScHe-panel as well as -pipes against critical heat loads coming from the plasma or ECRH stray radiation.

The ScHe-panel consists of six austenitic stainless steel (SS) tubes with a dimension of $12 \times 1 \mathrm{~mm}$. The tubes are welded pair-wise via a connection box and are fixed with a SS-bracket that is thermally isolated with a ceramic element against the ScHe-pipes. The ScHe thus flows in a meander arrangement (Fig. 3).

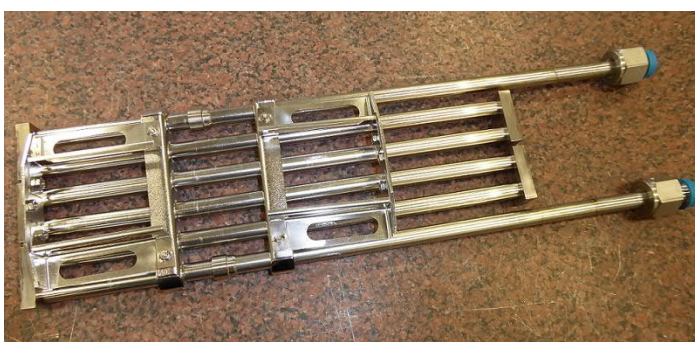

Fig. 3: ScHe-panel of the W7-X CVP

\section{2 $\mathrm{LN}_{2}$-reflector and $\mathrm{LN}_{2}$-chevron}

The $\mathrm{ScHe}$-panel is mounted to a surrounding $\mathrm{LN}_{2}$-cooled reflector (Fig. 4) by the SS-bracket structure as well. This $\mathrm{LN}_{2}$-reflector reflects and absorbs the radiant heat loads from the divertor chamber and leads to a reduction of the heat flow to the ScHe-panel. It consists of SS-plates which are TIG welded to the $\mathrm{LN}_{2}$-pipes. Fig. 4 shows the $\mathrm{LN}_{2}$-reflector before and after inert gas annealing that was performed to remove temper colors and undesirable residuals from the manufacturing process. Electrochemical cleaning, e.g pickling of surfaces that contain small (capillary) gaps where the cleansing agent could be trapped, is not allowed for ultra-high-vacuum (UHV) W7-X components. The remaining agent might lead to corrosion in conjunction with air humidity. Mechanical removal, by grinding or brushing, would be exceptionally time consuming and could not be performed 100\%successfully anyway. Inert gas annealing leads to a residue-free removal of temper colors. It was performed at a temperature of $950{ }^{\circ} \mathrm{C}-1100{ }^{\circ} \mathrm{C}$ for 5-20 min. with Argon or at other purposes with Hydrogen, at 250-350 mbar pressure in the glowing chamber.

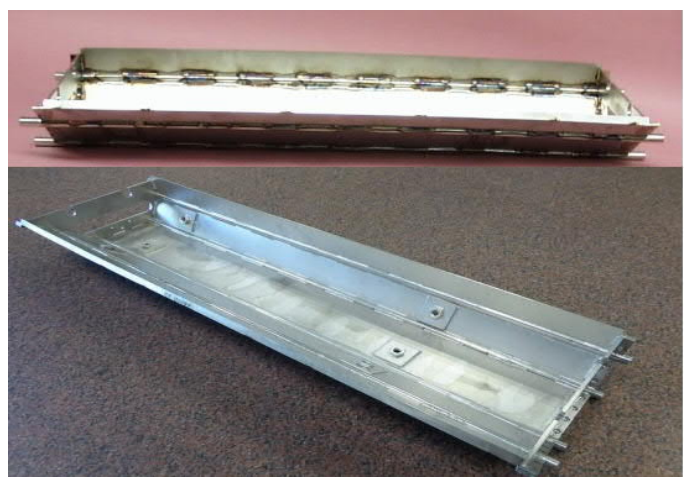

Fig. 4: $\mathrm{LN}_{2}$-reflector before (above) and after inert gas annealing

The ScHe-panel and $\mathrm{LN}_{2}$-reflector are joined together with a $\mathrm{LN}_{2}$-chevron completing the divertor-cryo vacuum pump-unit (DCU), see Fig. 5. The chevron covers the DCU against the pumping gap direction and still allows the pumped gases to pass into the DCU. The incoming infrared heat radiation is absorbed by a blackened rough surface of the chevron sheets. The chevron is made from angular SS-sheets welded to $\mathrm{LN}_{2}$-pipes. Welding gaps at the backside of these lamellas are closed and filled by hard-soldering in order to avoid feigned leaks and to improve heat transfer [5], s. Fig. 5. Sand blasting of the sheet metal surfaces was performed to ensure uniform spreading of the radiation. The blasted surfaces were then blackened by a black oxide finishing treatment by dipping 
the SS-sheets into an alkaline electrolyte bath. The Fecontent in the alloy was thereby changed at the top surface to mixed layers of iron oxide $\left(\mathrm{FeO}\right.$ and $\left.\mathrm{Fe}_{2} \mathrm{O}_{3}\right)$ which produced the black color. Concerning this transformation process the blackening was not a film-forming procedure. In this context, it was not applied to make the surface very robust against deformation or damage. The aim was to improve the absorption of the heat radiation.

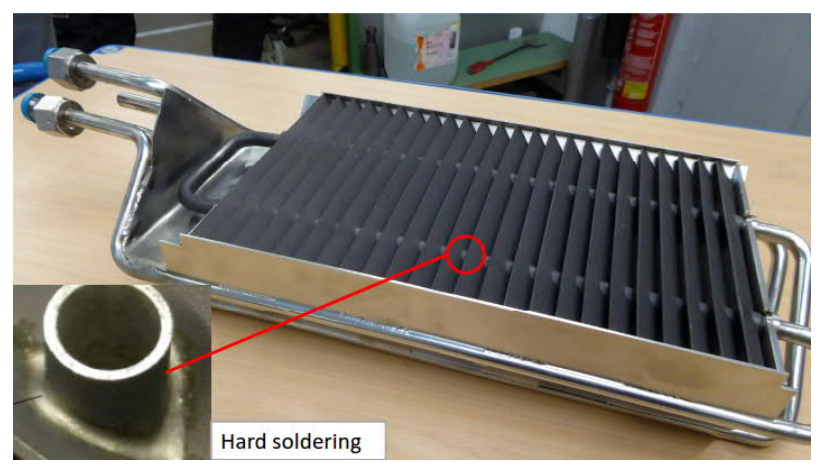

Fig. 5: $\mathrm{LN}_{2}$-chevron constitutes together with the ScHe-panel (not visible) and the $\mathrm{LN}_{2}$-reflector to a complete $\mathrm{DCU}$

With that technology it was achieved to come to an absorption coefficient $\varepsilon>0.6$ for infrared radiation, and 0.02 for microwave radiation (ECRH-heating). For the microwave absorption it is not far from a blank, nontreated stainless steel $(\varepsilon=0.01)$. This property was taken into account during the calculation of the CVP heat loads $(\S 4)$.

\subsection{DCUs}

The W7-X CVP consists of two DCUs, DCU1 and DCU2. The positions and dimensions of the units are defined by the locations of diagnostics, ports, pumping gaps and other divertor or in-vessel components. DCU1 and DCU2 are connected in series via transfer lines made of SSpipes, TIG-welded by hand to SS-radiation shields. The transfer lines contain the $\mathrm{ScHe}$ - and $\mathrm{LN}_{2}$-pipes. The TIGwelding along the full length caused an extensive rebending due to the distortions coming from the weld shrinkage. For later, similar welding tasks the situation was improved significantly by new and innovative processes like electron-beam welding in vacuum where the heat input and subsequently the amount of required rework was greatly reduced.

An actively cooled room temperature water baffle (RTchevron) was built in order to additionally reduce the impact of the plasma heat radiation onto the CVP (Fig. 6). One RT-chevron is positioned in front of each DCU, and connected in series via a transfer line as well. The heat shield of the RT-Chevron transfer line is built out of a SSground layer with $2 \mathrm{~mm}$ thickness for adequate stiffness which is plated with a $2 \mathrm{~mm}$ thick $\mathrm{Cu}$ layer for high heat conduction. The design of the RT-chevron is similar to the $\mathrm{LN}_{2}$-chevron of the DCUs. The SS-lamella are replaced by copper alloy, hard-soldered to the SS pipes, and are coated by a ceramic $\mathrm{Al}_{2} \mathrm{O}_{3}-13 \mathrm{TiO}_{2}$ layer produced with atmospheric plasma sputtering. The layer material has a thickness of 120-170 $\mu \mathrm{m}$ [7]. This layer is not comparable with the blackening of the $\mathrm{LN} 2$-chevron. The $\mathrm{Al}_{2} \mathrm{O}_{3}$ $13 \mathrm{TiO}_{2}$ is a sputtered film that could loosen in particular in areas where a multi layers film was applied. This has happened when the plasma gun, operating at $\sim 2300{ }^{\circ} \mathrm{C}$, had to coat the ceramic multiple times. That required a sensible handling of the RT-chevron during the assembly process, as well as permanent visual inspections of the adjacent components to avoid an input of loosened particles.

The CVP is connected via another transfer line to the "cryo-plugin", a feedthrough that transports the cryogenic media from outside to the CVP in the plasma vessel. Design and manufacturing of the plugin will be discussed in a future paper.

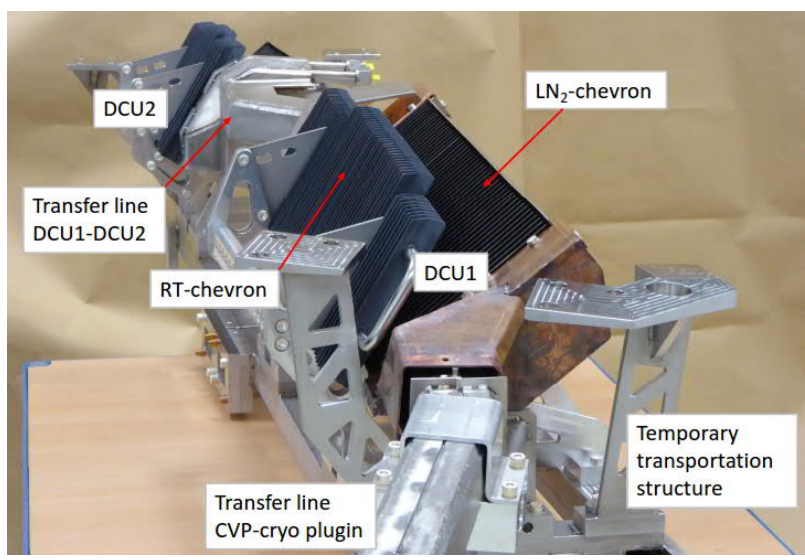

Fig. 6: completed CVP with temporary transportation structure (without transportation structure $2.6 \mathrm{~m}$ long and $60 \mathrm{~kg}$ heavy)

Originally, the RT-chevron was intended to intercept a heat load of $150 \mathrm{~kW} / \mathrm{m}^{2}$ plasma radiation. However, updated calculations showed a negligible amount of this power flow into the divertor chamber. On the other hand, the ECRH-stray radiation onto the in-vessel components and the plasma vessel itself was realized to be important. The RT-chevron, due to its high absorption coefficient for ECRH-stray radiation $\left(\sim 70 \%\right.$ from the $\mathrm{Al}_{2} \mathrm{O}_{3}-13 \mathrm{TiO}_{2}$ layer), is capable to reduce this radiation significantly from $38 \mathrm{~kW} / \mathrm{m}^{2}$ stray radiation load in the divertor chamber (for machine modules with the highest amount of ECRH-power) to $12 \mathrm{~kW} / \mathrm{m}^{2}$ at the RT-chevron backside.

\section{Heat load calculations and cryo supply}

However, the absorption of ECRH-stray radiation loads by the RT-chevron leads to an additional heating up of this chevron. Thus, the RT-chevron radiates with $1800 \mathrm{~kW} / \mathrm{m}^{2}$ to the $\mathrm{LN}_{2}$-chevron and causes there an exceeding of the allowable heat load on the $\mathrm{LN}_{2}$-circuits which is $1200 \mathrm{~W}$ per CVP $[8,9]$. Therefore, additional measures had to be implemented to avoid overheating of the cryo supply system under long pulse operations.

The first measure was to supply the overloaded CVPs in the W7-X modules with the highest ECRH-power with a higher $\mathrm{LN}_{2}$ flow rate by reducing the flow rate in $\mathrm{W} 7-\mathrm{X}$ modules with less ECRH-power [9]. Due to the parallel supply of the CVPs with $\mathrm{ScHe}$ and $\mathrm{LN}_{2}$ the cryo periphery outside the machine had to be changed to enable a inhomogeneous distribution of $\mathrm{LN}_{2}$. Secondly, an additional ECRH-shield ("ECRH-housing") was 
integrated in all CVPs to close the gap between the RTchevron and the $\mathrm{LN}_{2}$-chevron (Fig. 7). This shield is made of copper sheet and limits the gap to the other components to $1 \mathrm{~mm}$. Thus the ECRH stray radiation is quasi forced to access the CVP only by passing through the RTChevron followed by the $\mathrm{LN}_{2}$-chevron until it reaches the $\mathrm{ScHe}$-panel and the $\mathrm{LN}_{2}$-reflector.

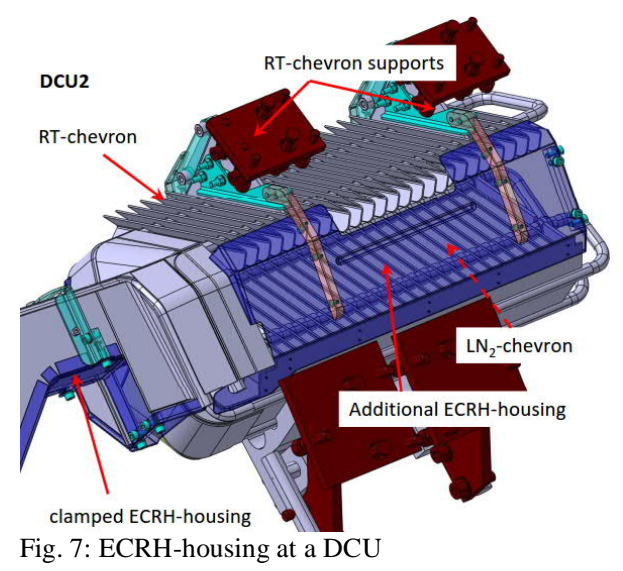

The stepwise reduction of heat loads towards the originally specified maximum input power of $1200 \mathrm{~W}$ per $\mathrm{LN}_{2}$-cirquit of the CVP $(\sim 16 \mathrm{~kW}$ for RT-chevron, $\sim 30 \mathrm{~W}$ for He-panel) was confirmed by extensive finite element analyses [8].

\section{Quality assurance and control}

Manufacturing of the CVP was monitored extensively by internal quality inspections at the IPP workshop as well as by the quality management department (QM) of W7-X. The internal inspections were guided by specified quality inspection plans and were divided into three main fields of activity:

1. Visual inspections concerning impurities, temper colours, welding quality, completeness and damages

2. Geometrical tests

3. He leak rate tests at different conditions

QM supported these inspections by a temporary external supervisor who was certified by state of the art industrial norms, e.g. for welding and X-Ray inspections. The weld seams were inspected with DIN EN ISO 5817-B which conforms to the highest applicable assessment level of weld seams.

On the other hand, the main focus of quality inspection was laid on hot and cryogenic integral Helium leak tests which were also the final approval of the CVPmanufacturing. The acceptance criteria were: maximal He-leakage rates of $5^{*} 10^{-8} \mathrm{mbar} * 1 / \mathrm{s}$ at $160{ }^{\circ} \mathrm{C}$ and $5^{*} 10^{-9}$ mbar*1/s at RT as well as at $77 \mathrm{~K}$ with $\mathrm{LN}_{2}$-cooling. The individual steps of these tests were:

1. Evacuate the vacuum test chamber with the installed CVP down to $10^{-5} \mathrm{mbar}$

2. He-leak test under 40 bar at RT, analyze the residual gas with a mass spectrometer (this was done also in the subsequent tests)
3. Cool down the CVP with $\mathrm{LN}_{2}$ to $77 \mathrm{~K}$ and $\mathrm{He}-$ leak test at 40 bar internal pressure $\left(\mathrm{LN}_{2}\right.$ is flushed out by the He; increase of CVP temperature by $\approx 3 \mathrm{~K}$ )

4. Heat up to $160{ }^{\circ} \mathrm{C}$ and He-leak test at 25 bar

5. Cool down to $<60{ }^{\circ} \mathrm{C}$ and He-leak test with 40 bar

6. Cool down to RT

7. Vent the vacuum test chamber

\section{Conclusions}

Manufacturing of the ten W7-X CVPs was finished in 2018 on schedule. The final work acceptance tests demonstrated that the CVPs were produced as specified. Due to their manufacture as welded sheet metal and pipe construction, the CVP caused a substantial amount of post-manufacturing processing like cleaning and rebending. The lesson learned from this was to improve the situation at similar tasks by applying newest manufacturing processes such as e.g. e-beam welding.

Assembly tests were already performed in a plasma vessel mockup on site. They confirmed that the CVP can be assembled together with the HHF targets within the extremely restricted space of the divertor chamber. By installing the additional ECRH shield, the heat impact onto the CVP can be reduced to an allowable level.

\section{References}

[1] T. Sunn Pedersen, A. Dinklage, Y. Turkin, R. Wolf, S. Bozhenkov, J. Geiger, G. Fuchert, H.S. Bosch, K. Rahbarnia, H. Thomsen, U. Neuner, W7-X Team, Key results from the first plasma operation phase and outlook for future performance in Wendelstein 7-X. Physics of Plasmas, 24, p.055503 (2017)

[2] H. Grote, J. Kisslinger, H. Renner, J. Boscary, H. Greuner, F.W. Hoffmann, B. Mendelevitch, Neutral particle modelling and particle exhaust in the Wendelstein 7-X stellarator, Journal of Nuclear Materials 313-316 (2003) 1298-1303

[3] A. Peacock, J. Boscary, M. Czerwinski, G. Ehrke, H. Greuner, P. Junghanns et. al., Status of High Heat Flux Components at W7-X, IEEE Trans. Plasma Sci. 42 (3) (2014) 524-532

[4] J. Boscary, G. Ehrke, H. Greuner, P. Junghanns, C. Li, B. Mendelevitch et. al., Progress in the Production of the W7$\mathrm{X}$ Target Modules, this conference

[5] B. Streibl, C. Li et. al., W7-X internal Ringbook for Cryo Vacuum Pump

[6] C. Day, Karlsruhe Institute of Technology (KIT), Institute of Technical Physics, Eggenstein-Leopoldshafen, Germany, internal report, Basics and applications of cryopumps

[7] A. Killinger, V. Martinez Garcia, A. Hoppmann, quality report of the $\mathrm{Al}_{2} \mathrm{O}_{3}-13 \mathrm{TiO}_{2}$ sputtering, NMI-TTI GmbH in collaboration with the Institut for manufacturing of ceramic components (IFKB) of the Univerity Stuttgart, Germany

[8] J. Zhu, V. Bykov, M. Nagel, G. Ehrke, J. Fellinger, L. Wegener, H-S. Bosch et. al., Refined Multiphysics Analysis of W7-XCryopumps, 2018 IEEE Trans. on Plasma Science 46 (5) 1592-602

[9] M. Nagel, M. Ihrke, M. Pietsch, H.-S. Bosch, C.P. Dhard and Th. Rummel, Concept for the cryo distribution for the Wendelstein 7-X cryo vacuum pumps, ICEC27-ICMC 2018 conference, Oxford, United Kingdom 